\title{
Atmospheres of Extra-solar Planets and Brown Dwarfs
}

\author{
France Allard \\ CRAL-ENS, 46 Allée d'Italie, 69364 Lyon Cedex 07, France
}

Travis S. Barman

Dept. of Physics \& Astronomy, Wichita State University, Wichita, KS 67226-0032, U.S.A.

\begin{abstract}
Brown dwarfs have masses intermediate to stars and planets, but their atmospheres can span planetary plasma conditions as they cool over time. They are more directly observable than the currently known extra-solar planets. In this paper, we provide a brief summary of the atmospheric properties of brown dwarfs, and apply this knowledge to predict the thermal and spectral properties of extra-solar planets.
\end{abstract}

\section{Introduction}

Since their first discovery in the Pleiades (Teide 1 by Rebolo et al. 1995) and in the solar neighborhood (Gliese 229B by Nakajima et al. 1996), brown dwarfs are found by the dozen mostly by photometric sky surveys (DENIS, 2MASS, SLOAN, etc.). Most brown dwarfs have been found within only $10 \mathrm{pc}$ (32.6 ly). For some, accurate distances can be established by the parallax method, and good resolution optical and near-infrared spectra are often obtained. Over the years, models of atmospheres and interiors have continued to improve in their ability to describe the global properties of brown dwarfs (Lunine et al. 1989; Burrows et al. 1993; Allard \& Hauschildt 1995; Tsuji et al. 1996; Chabrier et al. 2000; Allard et al. 2001). Consequently, brown dwarfs are fairly well understood and can, therefore, serve as stepping stones for modeling extra-solar planets.

\section{Brown Dwarfs}

Figure 1 summarizes what we have learned of the overall spectral properties of brown dwarfs from the stellar to the planetary temperature range. The topmost spectrum is that of a low mass $M$ dwarf $\operatorname{star}\left(M / M_{\odot}=0.1\right)$ which burns hydrogen in its core and will continue to do so for billions of years. Despite their ability to burn hydrogen, $\mathrm{M}$ dwarfs $\left(0.3 \geq \mathrm{M} / \mathrm{M}_{\odot} \geq 0.07\right)$ have low intrinsic luminosities $\left(10^{-2} \geq \mathrm{L} / \mathrm{L}_{\odot} \geq 10^{-4}\right)$ which lead to cool surface temperatures and strong molecular spectral features. Young brown dwarfs $(\leq 1 \mathrm{Gyr}$, such as those found in young stellar clusters and associations, may initially be as hot as $2800 \mathrm{~K}$, and have spectra similar to $\mathrm{M}$ dwarfs but with lower surface gravities $(\log g=3.5-4.5)$. Since brown dwarfs do not achieve stable hydrogen burning 

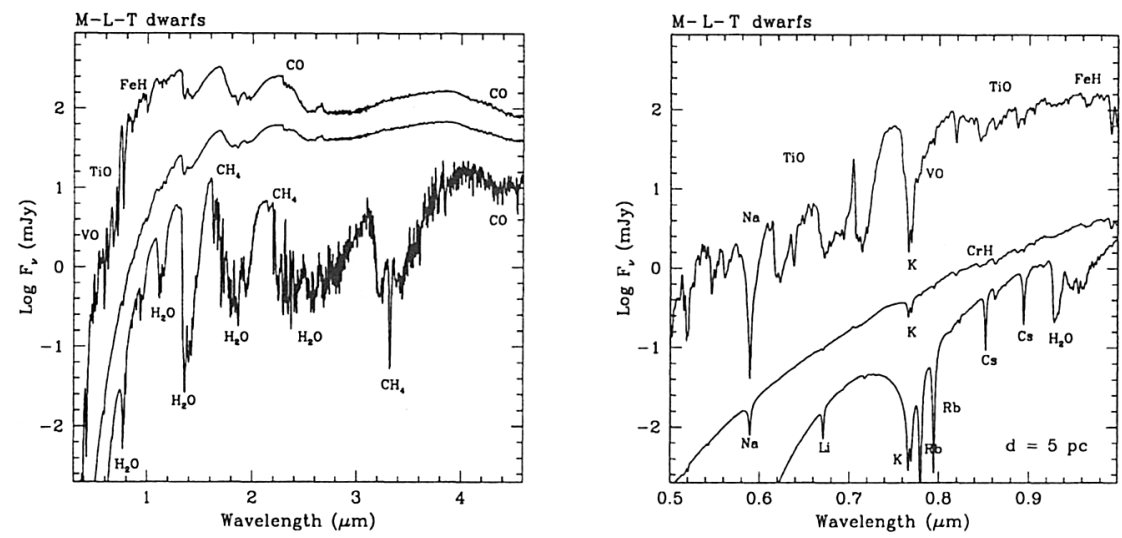

Figure 1. The Spectral Energy Distributions (SED) of a typical brown dwarf as it cools down over time through the $\mathrm{M}, \mathrm{L}$, and $\mathrm{T}$ spectral types according to models by Allard et al. (2001). The surface temperature (effective) from the top to bottom curve is 2500,1800 and $1000 \mathrm{~K}$ respectively, and surface gravities and radii are taken from Chabrier et al. (2000) for an age of 5 Gyr. These parameters are typical of brown dwarfs and low mass stars of the solar neighborhood. The spectral resolution has been reduced, and the most important molecular absorption bands are indicated for clarity. Iron and silicate dust grain formation warms and reddens the SED of L dwarfs while coolest $\mathrm{T}$ dwarfs have relative dust-free atmospheres.

in their core, they merely cool off as their luminosity diminishes with time, and they exist with a variety of temperatures and luminosities below these values.

The conditions in brown dwarf atmospheres can span between $\mathrm{T}_{\text {gas }}=80-3200 \mathrm{~K}, \rho=0.061-13.5 \mu \mathrm{g} / \mathrm{cm}^{3}$ and $\mathrm{P}_{\text {gas }}=1.75 \times 10^{-4}-1.65 \mathrm{~atm}$. The second spectrum (from the top) is that of an L-type brown dwarf, where the L-type indicates that $\mathrm{TiO}$ features begin to disappear from the optical spectrum, due to titanate $\left(\mathrm{CaTiO}_{3}, \mathrm{Ti}_{2} \mathrm{O}_{4}\right.$, etc. $)$ dust grain formation, while other spectral features are revealed ( $\mathrm{CrH}$ bands, Cs I lines). The redistribution of heat by silicate dust causes L-dwarfs to emit more near-infrared flux (relative to the optical) than earlier-type dwarfs. Indeed, at the prevailing atmospheric temperatures $\left(\mathrm{T}_{\text {gas }}<1600 \mathrm{~K}\right)$, strong absorbers, such as $\mathrm{MgSiO}_{3}$ and $\mathrm{Mg}_{2} \mathrm{SiO}_{4}$ condensates, begin to form. When brown dwarfs reach $1800 \mathrm{~K}$, as shown in Fig. 1, the effects of dust reach their maximum. Below $1700 \mathrm{~K}$, silicates form at layers which are too deep to affect the emerging spectrum. The spectrum of a $1000 \mathrm{~K}$, dust-free atmosphere is illustrated by the bottom spectrum.

We know, based upon solid observations, that the latest $\mathrm{M}$ and most $\mathrm{L}$ dwarfs carry atmospheric dust. But how can this dust remain suspended in these low luminosity atmospheres? Radiation pressure is negligible and is certainly not the answer. Because of the relatively inefficient transport of energy by radiation and the large molecular opacities, these cool atmospheres are all convective. It is convection that provides the essential replenishment of condensible material 

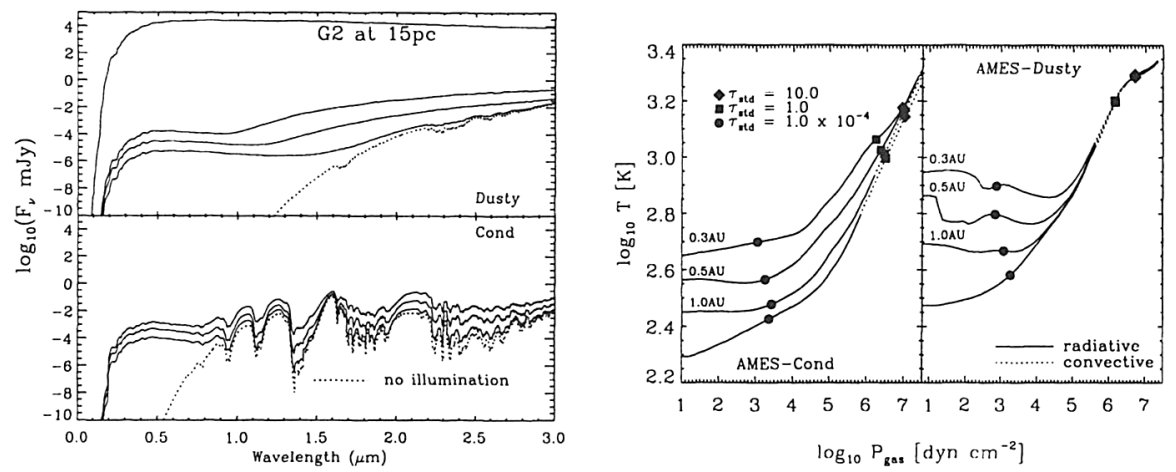

Figure 2. Spectra and thermal structures for a non-irradiated and irradiated $\left(\mathrm{T}_{\text {int }}=500 \mathrm{~K}, \log g=3.5\right)$ planet when located $1.0,0.5$ and $0.3 \mathrm{AU}$ from a G2 primary star. For comparison, the spectrum of the G2 star is also shown. All fluxes have been scaled appropriately for the size of the planet (or primary) and have been scaled to a distance of 15 parsecs. The filled symbols in the structure plot refer to different optical depths $(\tau)$ defined at $\lambda=1.2 \mu \mathrm{m}$.

in the cloud layers. We, therefore, understand the presence of dust as a delicate equilibrium between 1) the sedimentation of the largest grains by gravity to lower, hotter layers where they are melted and sublimated, 2) the convective updraft of condensible material, and 3) the efficiency of the condensation process. It is now clear that the cloud layer can only exist in close proximity to the top of the convection zone and, as the radiative-convective boundary retreats with decreasing luminosity, the cloud layer also sinks deeper into the atmosphere. Moreover, it is also possible that in parallel, the cloud surface coverage decreases (Burgasser et al. 2002). These reasons explain why Gliese 229B, a $900 \mathrm{~K}$ brown dwarf, is relatively dust-free, while all younger, hotter, and more massive brown dwarfs known are strongly affected by dust. Today, other such dust-free brown dwarfs are known, as well as intermediate cases. Such objects help to constrain the most recent models which include these physical principles (Ackerman \& Marley 2001; Allard et al. 2003).

\section{Extra-Solar Planets}

The discovery of the first extra-solar giant planet (EGP) was announced in the same year (1995) as the discovery of Gliese 229B. Since that time, the discovery rate of EGPs has only been slightly behind that of brown dwarfs; nearly 100 EGPs have been detected so far (compared to roughly 170 brown dwarfs). EGPs are, by definition, associated with a parent star which, in most cases so far, is similar to our Sun and have orbital separations which are very small compared to that of Jupiter. Their close proximity to a solar-type star makes the modeling of such objects slightly more complicated than brown dwarfs because both the intrinsic interior source of heat and the intense extrinsic solar radiation must be accounted for. 
Barman et al. (2001) have modeled EGPs at various orbital distances and cooling stages. Figure 2 demonstrates the effects of irradiation on the atmosphere of an EGP which has cooled internally to $500 \mathrm{~K}$. This corresponds to an age of about 10 million years (Baraffe et al. 2002). This particular case illustrates the dramatic heating that occurs in the atmosphere of EGPs which are found as close as $0.05 \mathrm{AU}$ from their parent star. The stellar radiation is capable of warming the planet's surface to above $1600 \mathrm{~K}$, which implies that conditions similar to dusty L-type brown dwarfs may prevail in these so called "hot jupiters". Therefore, we can expect to find magnesium silicate clouds at the surface of EGPs similar to those found in brown dwarfs.

Figure 2 also demonstrates the effects of irradiation for two extreme cases of condensation in an EGP atmosphere. In the right panel we compare the temperature structures for a cloud free (AMES-Cond) and cloudy (AMES-Dusty) planet located a various orbital separations. In the cloud free model, the stellar radiation penetrates deep into the atmosphere and heats the layers near the radiative-convective boundary. At small orbital separations, the heating is sufficient to suppress convection in most of the atmosphere. In the cloudy case, the dust effectively shields the inner layers of the atmosphere from the irradiation and heating only occurs at the top of the atmosphere. The spectra for each of these model atmospheres are shown in the left panel. The cloud free EGP atmospheres have spectra similar to the dust free brown dwarf shown in Fig. 1. However, as the planet is brought closer to the parent star, the molecular absorption features are diminished due to the flattening of the temperature profile. The dusty atmospheres have nearly featureless spectra due to the "grey" characteristics of dust opacity.

\section{References}

Ackerman, A. S., \& Marley, M. S. 2001, ApJ, 556, 872

Allard, F., Guillot, T., Ludwig, H.-G., \& Hauschildt, P. H. 2003, ApJ, accepted Allard, F. \& Hauschildt, P. H. 1995, ApJ, 445, 433

Allard, F., Hauschildt, P. H., Alexander, D. R., Tamanai, A., \& Schweitzer, A. 2001, ApJ, 556, 357

Baraffe, I., Chabrier, G., Allard, F. \& Hauschildt, P. H. 2002, A\&A, 382, 563

Barman, T. S., Hauschildt, P. H., \& Allard, F. 2001, ApJ, 556, 885

Burgasser, A. J., et al. 2002, ApJ, 571, L151

Burrows, A., Hubbard, W. B., Saumon, D., \& Lunine, J. I. 1993, ApJ, 406, 158

Chabrier, G., Baraffe, I., Allard, F., \& Hauschildt, P. H. 2000, ApJ, 542, 464

Lunine, J. I., Hubbard, W. B., Burrows, A., Wang, Y.-P., \& Garlow, K. 1989, ApJ, 338, L314

Nakajima, T., Oppenheimer, B. R., Kulkarni, S. R., Golimowski, D. A., Matthews, K., \& Durrance, S. T. 1995, Nature, 378, 463

Rebolo, R., Zapatero-Osorio, M. R., \& Martìn, E. L. 1995, Nature, 377, 129

Tsuji, T., Ohnaka, K., Aoki, W., \& Nakajima, T. 1996, ApJ, A\&A, 308, 29 